\title{
The ribose and glycine Maillard reaction in the interstellar medium (ISM): A theoretical study
}

\author{
ABRAHAM F JALBOUT ${ }^{\mathrm{a}, *}$ and MD ABUL HAIDER SHIPAR ${ }^{\mathrm{b}}$ \\ ${ }^{a}$ Instituto de Quimica, Universidad Nacional Autonoma de Mexico, Mexico City, Mexico \\ ${ }^{\mathrm{b}}$ Faculty of Engineering, Chiba University, Inage-ku, Chiba 263-8522, Japan \\ e-mail: ajalbout@u.arizona.edu
}

MS received 26 October 2007; revised 22 December 2007

\begin{abstract}
Possibility of the Maillard reaction to take place in the gaseous phase in the interstellar medium was investigated by using Density Functional Theory (DFT) computations. Cyclic ribose (c-Rib)/ open-chain ribose (c-Rib) and glycine were taken as the model. Mechanisms have been proposed, and possibility of the formation of different compounds have been evaluated through calculating the Gibb's free energy changes for different steps of the reaction by following the total mass balance. The result reveals that both c-Rib and Rib can participate in the reaction, and c-Rib is more efficient than Rib. The reactions under basic and neutral conditions are supposed to be the first and second most favourable. Acidic conditions and the isoelectric point of glycine were unfeasible for the reaction. The kinetics of the mechanics are briefly addressed in this work.
\end{abstract}

Keywords. Density functional computational study; ribose; glycine; Maillard reaction; gaseous phase interstellar medium.

\section{Introduction}

Non-enzymatic browning, also known as the Maillard reaction, ${ }^{1}$ occurs between carbonyl compounds, especially reducing sugars, and compounds with free amino groups, such as amines, amino acids, and proteins. The reaction in aqueous solution is usually vital to foods as well as to general biochemistry and even to medicine. ${ }^{2-5}$ The gaseous phase Maillard reaction has not been well studied, and therefore, the role of the reaction under this condition is obscure. It is evident from theoretical and experimental studies to form larger sugars, such as ribose, from simple organic precursors in the gaseous phase ${ }^{6,7}$ However, no clear-cut evidence is still available. Ribose might have played an important role during the creation of the first living organism, could be in the gaseous phase interstellar medium, in the early history of earth or in the 'pre-RNA or RNA-world' ${ }^{8,9}$ Ribose can be formed from simple organic precursors, formaldehyde and glycolaldehyde ${ }^{10}$ Formaldehyde is a significantly abundant interstellar molecule, and glycoaldehyde has been detected in the interstellar medium. ${ }^{11}$ Therefore, 'RNA world' is assumed to have a large

*For correspondence pre-biotic source of ribose, formed through the 'formose reaction' of formaldehyde and glycolaldehyde..$^{10,11}$

Meteorites impact on Earth have been shown to carry many amino acids. ${ }^{12}$ The amino acid, glycine, might have been detected in the gaseous phase in the interstellar medium. ${ }^{13}$ However, this report has recently been challenged. ${ }^{14}$ Blagojevic et al have proposed a possible ion-molecule reaction pathway to interstellar amino acid synthesis. ${ }^{15}$ Jackson et $a l^{16}$ have investigated the possibility of forming glycine from interstellar species through the ion-molecule reactions of ionized amine fragment compounds with neutral carboxylic acid and ester species. By taking these findings in mind, we can assume that ribose (from formaldehyde and glycolaldehyde) and glycine (from simple atmospherically abundant molecules) can be formed in the gaseous phase in the interstellar medium, and therefore, the Maillard reaction between ribose and glycine can take place under this condition. While bulk solvation effects have not been taken into consideration we believe that the scarce nature of the free solvents in the ISM will prevent such conditions from affecting the outcome. However, test calculations by our group reveal that such effects shall account statistically for less than $5 \%$ of ISM mediated mechanism. 
It is well known that organic molecules comprise more than $75 \%$ of known interstellar chemical species. $^{2}$ Therefore, a variety of gas phase interstellar reactions dominated by two-body, exothermic, ionmolecule reactions permit reactions that lead to synthesis of larger molecules in the interstellar medium (up to 12 atoms). There are many atmospherically abundant molecules, including molecular hydrogen $\left(\mathrm{H}_{2}\right), \mathrm{H}_{3}^{+}$, and $\mathrm{H}_{3} \mathrm{O}^{+}\left(\mathrm{H}_{2}\right.$ is the most abundant interstellar chemical species) suggesting protonation may be an important ion-molecule reaction. Several different reaction conditions can be applied to catalyse the effective transformation of formaldehyde to ribose (Simonov et al 2007). There are many possible routes for this mechanism, among which $\alpha$-hydroxy ketones can catalyse the reaction.

Due to the fact that formaldehyde $\left(\mathrm{CH}_{2}=\mathrm{O}\right)$ is significantly abundant amongst the $\sim 140$ known interstellar molecules as well as the fact that protonated formaldehyde $\left(\mathrm{CH}_{2}=\mathrm{OH}^{+}\right)$has been detected we can use this to focus on similar reactions. In these experiments with formaldehyde ${ }^{7}$ a gas phase protic variant of the formose reaction is generally explored at 2 mbar using hydronium ion $\left(\mathrm{H}_{3} \mathrm{O}^{+}\right)$as a proton donating source to formaldehyde. Therefore, by using gas-phase conditions of standard temperature and pressure with zero-point corrections we can develop a simulated environment of the ISM.

A primary motivation for this research involves the fact that even mechanisms with high reaction barriers can potentially lead to interesting experimentally viable biological products. ${ }^{7}$ While the barriers observed are present (in the order of 50$60 \mathrm{~kJ} / \mathrm{mol}$ ) it must be noted that errors in the calculation exist. These computational faults lie in the order of $7-10 \mathrm{kcal} / \mathrm{mol}$ which in some cases would lead to a barrier-less chemical process. Experimentally important ISM product has been shown to form from the scheme we proposed and from further ISM observations we believe it to be a viable starting point for our model calculations.

In the present study, Density Functional Theory (DFT) computations have been applied to investigate possibility of the formation of the Amadori rearrangement products (ARPs) in the Maillard reaction between ribose and glycine in the gaseous phase. Mechanisms (figures 1 and 2) are proposed for the formation of ARPs in ribose + glycine Maillard reaction, followed usually the most accepted Hodgescheme. ${ }^{17}$ Ribose can exist in its open and closed chain or cyclic forms (Rib and c-Rib, respectively), ${ }^{3,4,8,18,19}$ and therefore, both of these forms of ribose have been considered in the present study to evaluate their activities in the reaction. Four forms of glycine (Gly), i.e. unionized or unprotonated glycine (UGly, $\mathrm{H}_{2} \mathrm{~N}-\mathrm{CH}_{2}-\mathrm{CO}_{2} \mathrm{H}$ ), protonated glycine (PGly, $\mathrm{H}_{3} \mathrm{~N}^{+}-\mathrm{CH}_{2}-\mathrm{CO}_{2} \mathrm{H}$ ), deprotonated glycine (DGly, $\mathrm{H}_{2} \mathrm{~N}-\mathrm{CH}_{2}-\mathrm{CO}_{2}:^{-}$) and glycine zwitterion (GlyZ, $\mathrm{H}_{3} \mathrm{~N}^{+}-\mathrm{CH}_{2}-\mathrm{CO}_{2}:^{-}$) under neutral $(\mathrm{pH}=7$ ), acidic $(\mathrm{pH}<5.5)$, and basic $(\mathrm{pH}>8)$ conditions and at the isoelectric point of glycine $(I=\mathrm{pH} \approx 6)$, respectively, ${ }^{3,4,18,19}$ are considered in the present study.

\section{Computational methodology}

All compounds in the proposed mechanisms (figures 1 and 2) have been optimized at RB3LYP/6-31G $(d)$ in GAUSSIAN 98 program. ${ }^{20}$ All RB3LYP/6-31G $(d)$ optimized structures have been taken for RB3LYP/ 6-31G $(d)$ frequency calculations to achieve the relevant zero point energy (ZPE) and the Gibb's free energy $\left(G^{\circ}\right)$ in the gaseous phase. ${ }^{21} \mathrm{Opt}=\mathrm{Z}$-matrix and/or Freq $=$ No Raman keywords have been used when necessary. ${ }^{21}$ By using RB3LYP/6-31G $(d)$ on the RB3LYP/6-31G $(d)$ optimized structures, relevant electronic energies in the gaseous have been

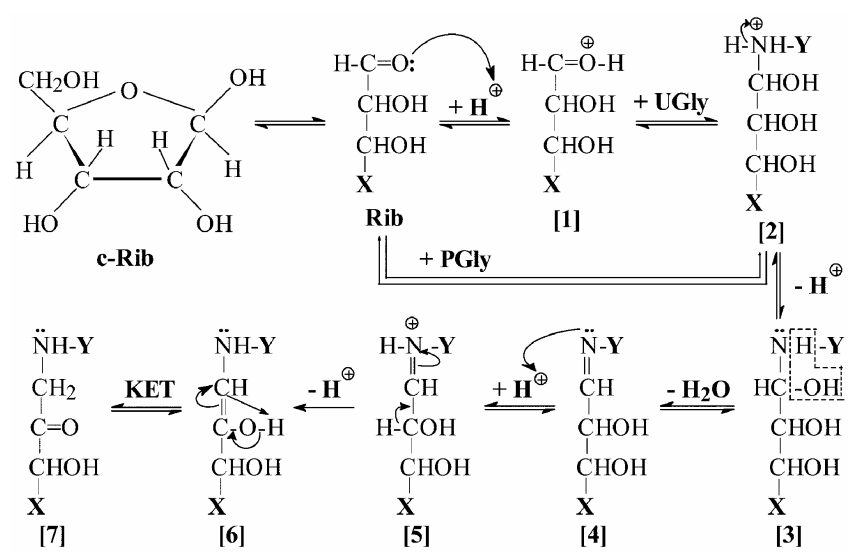

Figure 1. Proposed mechanisms for the formation of the Amadori rearrangement products in c-Rib + UGly/Rib + UGly and c-Rib + PGly/Rib + PGly Maillard reactions. Abbreviations: $\mathbf{c}-\mathbf{R i b}=$ cyclic ribose; $\mathbf{R i b}=$ open-chain ribose KET $=$ keto-enolic tautomerization. Numerical abbreviations: $\mathbf{1}=$ protonated form of Rib; 2 = ionic addition adduct of Rib and UGly or PGly (1-protonated glycino-pent-1,2,3,4,5-ol); $\mathbf{3}=$ addition compound of Rib and UGly or PGly (1-glycino-pent$1,2,3,4,5-\mathrm{ol}) ; \mathbf{4}=$ Schiff base of $\mathbf{3} ; \mathbf{5}=$ ionic adduct of $\mathbf{4}$; $\mathbf{6}=$ enol form of the Amadori rearrangement product (1glycino-pent-2,3,4,5-ol-1-ene); $7=$ keto form of the Amadori rearrangement product (1-glycino-pent-3,4,5-ol2-one). $\mathbf{X}$ and $\mathbf{Y}$ refer the $-\mathrm{CH}(\mathrm{OH})-\mathrm{CH}_{2} \mathrm{OH}$ and $-\mathrm{CH}_{2}-$ $\mathrm{CO}_{2} \mathrm{H}$ groups, respectively. For other abbreviations, see the caption of figure 1 . 
calculated. ${ }^{21}$ Relevant ZPE have been added to the electronic energies to get the total electronic energies $\left(E^{\circ}\right){ }^{21}$

Finally, electronic and free energy changes $\left(\Delta E^{\circ}=E_{\text {Product(s) }}^{\circ}-E_{\text {Reactant(s) }}^{\circ}\right.$, and $\Delta G^{\circ}=G_{\text {Product(s) }}^{\circ}-$

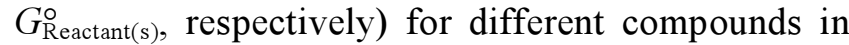
the proposed mechanisms have been calculated by following the total mass balance of the reaction. Thus, the possibility and internal energy changes for the formation of different compounds in the proposed mechanisms have been investigated. Relevant dipole moments $\left(\mu_{\text {Gas }}\right.$, in Debye) of different compounds in the gaseous phase have been obtained from the electronic energy calculations on the RB3LYP/6-31G(d) optimized structures at RB3LYP/6-31G $(d){ }^{21}$ Relevant heats of formation $\left(\Delta H_{f}^{\circ}\right)$ of different compounds have been obtained by performing AM1 semi-imperical calculations ${ }^{21}$ on the RB3LYP/6-31G(d) optimized structures.

\section{Results and discussion}

\subsection{Thermochemistry}

At constant temperatures and pressures, $\Delta E$ and $\Delta G$ of a reaction indicate the internal energy changes

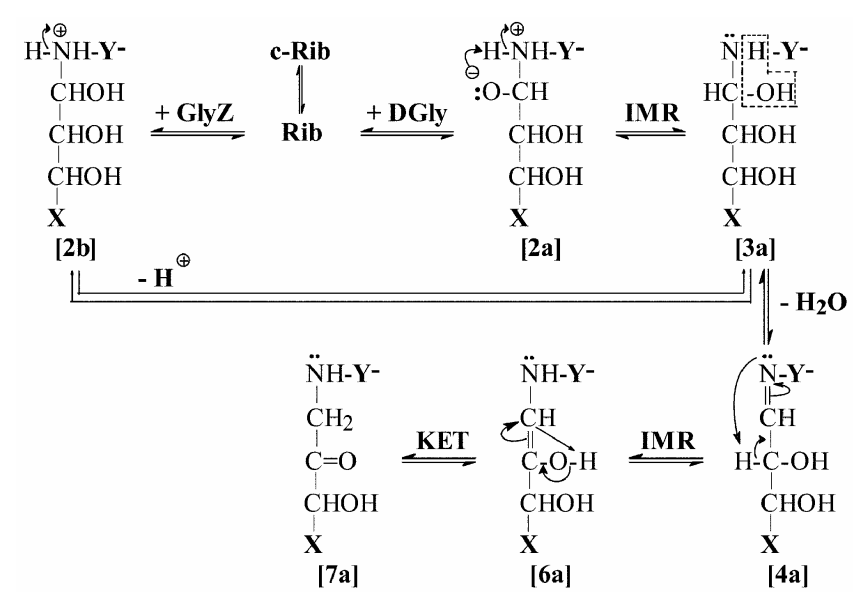

Figure 2. Proposed mechanisms for the formation of the Amadori rearrangement products in c-Rib + DGly/Rib + DGly and c-Rib + GlyZ/Rib + GlyZ Maillard reactions. Abbreviations: $\mathbf{I M R}=$ intramolecular rearrangement. Numerical abbreviations: $\mathbf{2 a}=$ ionic addition adduct of Rib and DGly; $\mathbf{2 b}=$ ionic addition adduct of Rib and GlyZ; 3a = addition compound of Rib and DGly or GlyZ (1-deprotonated glycino-pent-1,2,3,4,5-ol); $\mathbf{4 a}=$ Schiff base of $3 \mathbf{a} ; \mathbf{6 a}=$ enol form of the Amadori rearrangement product (1-deprotonated glycino-pent-2,3,4,5ol-1-ene); 7a = keto form of the Amadori rearrangement product (1-deprotonated glycino-pent-3,4,5-ol-2-one). $\mathbf{Y}^{-}$ Refers the $-\mathrm{CH}_{2}-\mathrm{CO}_{2}$ : $^{-}$group. For other abbreviations, see the caption of figures 1 and 2 . and spontaneity of the reaction, respectively. $\Delta G^{\mathrm{o}}$ for the formation of different compounds in the proposed mechanisms for c-Rib + Gly (Gly refers UGly, PGly, DGly and GlyZ) and Rib + Gly reactions (figures 1 and 2) are presented in table 1. During calculating $\Delta G^{\mathbf{o}}$, $\mathbf{c}-\mathbf{R i b}+\mathbf{G l y}$ and $\mathbf{R i b}+\mathbf{G l y}$ total free energies $\left(G_{\mathrm{c}-\mathrm{Rib}}^{\circ}+G_{\mathrm{UGly} / \mathrm{PGly} / \mathrm{DGly} / \mathrm{Glyz}}^{\circ}\right.$ and $G_{\mathrm{Rib}}^{\circ}+$

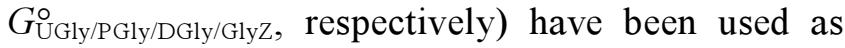
the standard in the equation $\Delta G^{\circ}=G_{\text {Product(s) }}^{\circ}-\mathrm{G}_{\text {Reactant(s) }}^{\circ}$. Table 1 also represents $\Delta E^{\circ}$ for the formation of different compounds in the proposed mechanisms (figures $\mathbf{l}$ and 2), obtained by using $\mathbf{c - R i b}+\mathbf{G l y}$ and Rib + Gly total electronic energies $\left(E_{\mathrm{c}-\mathrm{Rib}}^{\circ}+E_{\mathrm{UGly} / \mathrm{PGly} / \mathrm{DGly} / \mathrm{GlyZ}}^{\circ}\right.$

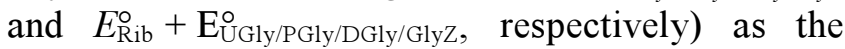
standard in the equation $\Delta E^{\circ}=E_{\text {Product(s) }}^{\circ}-E_{\text {Reactant(s). }}^{\circ}$. Dipole moments (in Debye) and $\Delta H_{\mathrm{f}}^{\circ}$ (in $\mathrm{kJ} / \mathrm{mol}$ ) for different compounds in the proposed mechanisms (figures 1 and 2) are also presented in table 1. Table 2 represents some RB3LYP/6-31G(d) geometric parameters of different compounds presented in figures 1 and 2 .

During the calculation of $\Delta G^{\circ}$ and $\Delta E^{\circ}$, the total mass balance of the reaction under different conditions has been maintained. The main problem in balancing the total mass arises for protonation and deprotonation. Molecular hydrogen $\left(\mathrm{H}_{2}\right), \mathrm{H}_{3}^{+}, \mathrm{H}_{3} \mathrm{O}^{+}$ etc. are present in the interstellar medium. ${ }^{22}$ Therefore, the following equation has been applied during balancing the total mass:

$$
\underset{\text { Proton }}{\mathrm{H}^{+}}+\underset{\text { water }}{\mathrm{H}_{2} \mathrm{O}} \stackrel{\leftrightarrow}{\leftrightarrow} \underset{\text { hydroxonium ion }}{\mathrm{H}_{3} \mathrm{O}^{+}}
$$

According to $\Delta G^{\circ}$ (table 1), production of Rib from c-Rib is probable in c-Rib + UGly and cRib + PGly reactions, and not in c-Rib + DGly and c-Rib + GlyZ reactions. Possibility of producing Rib from c-Rib in c-Rib + UGly and c-Rib + PGly reactions is supposed to be almost same (table 1). Based on $\Delta E^{\mathrm{o}}$ (table 1), Rib is electronically more stable in c-Rib + UGly reaction than that of the others. Based on $\Delta G^{\circ}$, protonation of Rib in c-Rib + UGly reaction is found to be more favourable than $\mathbf{R i b}+$ UGly reaction (table 1). Protonated ribose (1), formed in c-Rib + UGly reaction, is electronically more stable than that of $\mathbf{R i b}+\mathbf{U G l y}$ reaction.

Based on $\Delta G^{\circ}$ (table 1), c-Rib + UGly and Rib + UGly reactions are found to be feasible for the formation of the ionic addition adduct, 2 (1protonated glycino-pent-1,2,3,4,5-ol), ionic adduct of the Schiff base, 5 (ionic adduct of the Schiff base 
Table 1. $\Delta G_{\mathrm{gas}}^{\circ}, \Delta E_{\mathrm{gas}}^{\circ}, \mu_{\mathrm{Gas}}$, and $\Delta H_{\mathrm{f}}^{\circ}$ for different compounds presented in figures 1 and 2 .

\begin{tabular}{|c|c|c|c|c|c|c|c|c|c|c|}
\hline \multirow[b]{2}{*}{ Compounds $\downarrow$} & \multicolumn{4}{|c|}{$\Delta \mathrm{G}_{\mathrm{Gas}}^{\circ}(\mathrm{kJ} / \mathrm{mol})$} & \multicolumn{4}{|c|}{$\Delta E_{\text {Gas }}^{\circ}(\mathrm{kJ} / \mathrm{mol})$} & \multirow[b]{2}{*}{$\begin{array}{c}\mu_{\mathrm{Gas}} \\
\text { (Debye) }\end{array}$} & \multirow[b]{2}{*}{$\underset{(\mathrm{kJ} / \mathrm{mol})}{\Delta H_{\mathrm{f}}^{\circ}}$} \\
\hline & $\begin{array}{c}\text { c-Rib + } \\
\text { UGly }\end{array}$ & $\begin{array}{l}\text { Rib + } \\
\text { UGly }\end{array}$ & $\begin{array}{c}\text { c-Rib + } \\
\text { PGly }\end{array}$ & $\begin{array}{c}\text { Rib + } \\
\text { PGly }\end{array}$ & $\begin{array}{c}\text { c-Rib + } \\
\text { UGly }\end{array}$ & $\begin{array}{l}\text { Rib + } \\
\text { UGly }\end{array}$ & $\begin{array}{c}\text { c-Rib + } \\
\text { PGly }\end{array}$ & $\begin{array}{c}\text { Rib + } \\
\text { PGly }\end{array}$ & & \\
\hline Rib & $-21 \cdot 3$ & - & $-21 \cdot 28$ & - & $-22 \cdot 66$ & - & $-22 \cdot 6$ & - & $2 \cdot 0969$ & $-979 \cdot 4$ \\
\hline 1 & $-94 \cdot 3$ & $-73 \cdot 0$ & - & - & $-101 \cdot 7$ & $-79 \cdot 2$ & - & - & $7 \cdot 261$ & $-264 \cdot 4$ \\
\hline 2 & $-167 \cdot 9$ & $-146 \cdot 6$ & $45 \cdot 5$ & $66 \cdot 9$ & $-222 \cdot 4$ & $-199 \cdot 8$ & $-7 \cdot 9$ & $14 \cdot 6$ & $7 \cdot 4653$ & $-788 \cdot 4$ \\
\hline 3 & $37 \cdot 8$ & $59 \cdot 0$ & $251 \cdot 3$ & $272 \cdot 6$ & $-11 \cdot 1$ & $11 \cdot 5$ & $203 \cdot 4$ & $225 \cdot 9$ & 3.8739 & $-1381 \cdot 9$ \\
\hline 4 & $4 \cdot 3$ & $25 \cdot 6$ & $217 \cdot 8$ & $239 \cdot 1$ & -0.5 & $22 \cdot 1$ & $214 \cdot 0$ & $236 \cdot 6$ & $2 \cdot 0875$ & $-1105 \cdot 8$ \\
\hline 5 & $-181 \cdot 9$ & $-160 \cdot 6$ & 31.6 & $52 \cdot 9$ & $-193 \cdot 5$ & $-170 \cdot 9$ & 21.0 & 43.6 & $4 \cdot 2938$ & -515.9 \\
\hline 6 & $25 \cdot 3$ & $46 \cdot 6$ & $238 \cdot 8$ & $260 \cdot 1$ & $13 \cdot 2$ & $35 \cdot 8$ & $227 \cdot 7$ & $250 \cdot 3$ & $3 \cdot 3578$ & $-1136 \cdot 0$ \\
\hline 7 & $-32 \cdot 7$ & $-11 \cdot 4$ & $180 \cdot 9$ & $202 \cdot 1$ & $-40 \cdot 7$ & $-18 \cdot 1$ & $173 \cdot 8$ & $196 \cdot 3$ & $1 \cdot 0744$ & $-1149 \cdot 5$ \\
\hline Compounds $\downarrow$ & $\begin{array}{c}\text { c-Rib + } \\
\text { DGly }\end{array}$ & $\begin{array}{l}\text { Rib + } \\
\text { DGly }\end{array}$ & $\begin{array}{c}\text { c-Rib + } \\
\text { GlyZ }\end{array}$ & $\begin{array}{l}\text { Rib + } \\
\text { PGly }\end{array}$ & $\begin{array}{c}\text { c-Rib + } \\
\text { UGly }\end{array}$ & $\begin{array}{l}\text { Rib + } \\
\text { UGly }\end{array}$ & $\begin{array}{c}\text { c-Rib + } \\
\text { GlyZ }\end{array}$ & $\begin{array}{r}\text { Rib + } \\
\text { GlyZ }\end{array}$ & - & - \\
\hline Rib & $7 \cdot 63$ & - & $7 \cdot 72$ & - & $-22 \cdot 5$ & - & $-22 \cdot 41$ & - & - & - \\
\hline $2 a$ & $43 \cdot 3$ & $64 \cdot 6$ & - & - & $-27 \cdot 9$ & $-5 \cdot 3$ & - & - & $4 \cdot 2724$ & $-1511 \cdot 5$ \\
\hline $2 b$ & - & - & $46 \cdot 9$ & $68 \cdot 2$ & - & - & $-0 \cdot 7$ & $21 \cdot 9$ & $8 \cdot 5114$ & $-1296 \cdot 6$ \\
\hline $3 \mathbf{a}$ & $42 \cdot 4$ & $63 \cdot 7$ & $768 \cdot 1$ & 789.4 & $-28 \cdot 8$ & $-6 \cdot 2$ & $623 \cdot 3$ & 645.9 & 7.9653 & $-1590 \cdot 7$ \\
\hline $4 a$ & -109.7 & $-88 \cdot 4$ & $616 \cdot 0$ & $637 \cdot 3$ & $-96 \cdot 4$ & $-73 \cdot 8$ & $602 \cdot 2$ & $624 \cdot 7$ & $8 \cdot 8492$ & $-1220 \cdot 6$ \\
\hline $6 a$ & $-127 \cdot 8$ & $-106 \cdot 6$ & 597.8 & $619 \cdot 1$ & $-112 \cdot 9$ & $-90 \cdot 3$ & $585 \cdot 6$ & $608 \cdot 2$ & 9.0894 & $-1247 \cdot 3$ \\
\hline $7 \mathbf{a}$ & $-122 \cdot 1$ & $-100 \cdot 8$ & $603 \cdot 6$ & $624 \cdot 9$ & $-102 \cdot 3$ & -79.7 & $596 \cdot 2$ & 618.8 & 11.3309 & $-1251 \cdot 5$ \\
\hline
\end{tabular}

of 1-glycino-pent-1,2,3,4,5-ol), and the keto form of the Amadori rearrangement product, 7 (1-glycinopent-3,4,5-ol-2-one). The reactions are found not to be feasible for the formation of the addition compound, 3 (1-glycino-pent-1,2,3,4,5-ol), the Schiff base, 4 (Schiff base of 1-glycino-pent-1,2,3,4,5-ol), and the enol form of the Amadori rearrangement product, 6 (1-glycino-pent-2,3,4,5-ol-1-ene). Therefore, it is assumed that the consequently formed ionic addition adduct, 2 (figure 1), may directly eliminate one molecule of water, leading to the formation of ionic adduct of the Schiff base, $\mathbf{5}$, and deprotonation and ketoenolic tautomerization (KET) of $\mathbf{5}$ may take place at the same time to produce 7. c-Rib + UGly reaction is assumed more feasible for producing different compounds than Rib + UGly reaction. Different compounds, produced in c-Rib + UGly reaction, are found electronically more stable than that of Rib + UGly reaction $\left(\Delta E^{\circ}\right.$ in table 1$)$.

Except producing Rib from c-Rib in c-Rib + PGly reaction, formation of all other compounds in the proposed mechanisms for c-Rib + PGly and Rib + PGly reactions (figure 1 and $\Delta G^{\circ}$ in table 1). c-Rib + GlyZ and Rib + GlyZ reactions are not found to be feasible for producing all compounds in the proposed mechanism (figure 2 and $\Delta G^{\circ}$ in table 1). Compounds formed in c-Rib + GlyZ and Rib + GlyZ reactions are assumed electronically less sta- ble than compounds formed in c-Rib + PGly and Rib + PGly reactions $\left(\Delta E^{\circ}\right.$ in table 1$)$.

c-Rib + DGly and Rib + DGly reactions are not feasible for the formation of the ionic addition adduct, $\mathbf{2 a}$, and the addition compound, 3a (1-deprotonated glycino-pent-1,2,3,4,5-ol) (figure 2 and $\Delta G^{\circ}$ in table 1). The reactions are feasible for forming the Schiff base, 4a (Schiff base of 1-deprotonated glycinopent-1,2,3,4,5-ol), the enol form of the Amadori rearrangement product, 6a (1-deprotonated glycinopent-2,3,4,5-ol-1-ene), and the keto form of the Amadori rearrangement product, 7a (1-deprotonated glycino-pent-3,4,5-ol-2-one) (figure 2 and $\Delta G^{\circ}$ in table 1). Therefore, it is assumed that nucleophilic addition of $\mathbf{c - R i b} / \mathbf{R i b}$ and DGly and elimination of one molecule of water may take place at the same time, leading to the formation of the Schiff base, $\mathbf{4 a}$, which can produce 6a and $7 \mathbf{a}$ accordingly (figure 2). In producing different compounds, c-Rib + DGly reaction is assumed more favourable than Rib + DGly reaction. c-Rib + DGly and Rib + DGly reactions are more feasible for the formation of $\mathbf{6 a}$ and $7 \mathbf{a}$ than forming 6 and 7 in c-Rib + UGly and Rib + UGly reactions (figures 1 and 2 , and $\Delta G^{\circ}$ in table 1 ). c-Rib/Rib + DGly reaction facilitates formation of both of $\mathbf{6 a}$ and $\mathbf{7 a}$, whereas c-Rib/Rib + UGly reaction facilitates only formation of $7\left(\Delta G^{\circ}\right.$ in table 1$)$. Therefore, it is assumed that the rate of the reaction 
Table 2. Some RB3LYP/6-31G(d) geometric parameters of different compounds presented in figures 1 and 2.

\begin{tabular}{|c|c|c|c|c|c|c|c|c|c|c|c|}
\hline \multirow[b]{2}{*}{ Compounds $\downarrow$} & \multicolumn{6}{|c|}{ Bond lengths $(\AA)$} & \multicolumn{3}{|c|}{ Bond angles $\left(^{\circ}\right)$} & \multicolumn{2}{|c|}{ Dihedral angles $\left(^{\circ}\right)$} \\
\hline & $\mathrm{C}-\mathrm{C}$ & $\mathrm{C}-\mathrm{O}$ & $\mathrm{O}-\mathrm{H}$ & $\mathrm{C}-\mathrm{H}$ & $\mathrm{N}-\mathrm{C}$ & $\mathrm{N}-\mathrm{H}$ & $\mathrm{C}-\mathrm{C}-\mathrm{C}$ & $\mathrm{H}-\mathrm{C}-\mathrm{O}$ & $\mathrm{C}-\mathrm{N}-\mathrm{C}^{\mathrm{e}}$ & $\mathrm{C}-\mathrm{C}-\mathrm{C}-\mathrm{C}^{\mathrm{f}}$ & $\mathrm{C}-\mathrm{C}-\mathrm{N}-\mathrm{C}^{\mathrm{g}}$ \\
\hline c-Rib & $\begin{array}{l}1.544, \\
1.5367, \\
1.5468, \\
1.5235\end{array}$ & $\begin{array}{l}1.3882 \\
1.4028 \\
1.4301\end{array}$ & $\begin{array}{l}0.9724 \\
0.9737 \\
0.9686 \\
0.9687\end{array}$ & $\begin{array}{l}1.0979, \\
1.0984, \\
1.0983, \\
1.0999\end{array}$ & - & - & $\begin{array}{l}101 \cdot 9 \\
102 \cdot 9 \\
113 \cdot 5\end{array}$ & - & - & $-31 \cdot 8$ & - \\
\hline Rib & $\begin{array}{l}1 \cdot 5152 \\
1 \cdot 5542\end{array}$ & $1 \cdot 215^{\mathrm{b}}$ & & $1 \cdot 1075$ & - & - & $114 \cdot 3$ & $121 \cdot 6$ & - & $84 \cdot 3$ & - \\
\hline 1 & $\begin{array}{l}1.5384 \\
1.5479\end{array}$ & $1 \cdot 3599^{\mathrm{b}}$ & 0.975 & $1 \cdot 1209$ & - & - & $109 \cdot 6$ & $117 \cdot 8$ & - & 68 & - \\
\hline 2 & 1.5546 & - & - & - & $\begin{array}{l}1.5381, \\
1.4827^{d}\end{array}$ & $\begin{array}{l}1.0333, \\
1.0262\end{array}$ & $108 \cdot 6$ & - & $112 \cdot 8$ & $-172 \cdot 3$ & $-84 \cdot 8$ \\
\hline $\mathbf{2 a}$ & $1 \cdot 5669$ & $1 \cdot 2952$ & - & $1 \cdot 1303$ & $\begin{array}{l}1 \cdot 6177, \\
1 \cdot 47^{\mathrm{d}}\end{array}$ & $\begin{array}{l}1.0167, \\
1.0218\end{array}$ & $116 \cdot 2$ & 117 & $114 \cdot 2$ & $-61 \cdot 7$ & $133 \cdot 4$ \\
\hline $2 b$ & $1 \cdot 5544$ & - & - & - & $\begin{array}{l}1.5195 \\
1.4872^{\mathrm{d}}\end{array}$ & $\begin{array}{l}1.0197 \\
1.0278\end{array}$ & $111 \cdot 4$ & - & $111 \cdot 4$ & -75 & $147 \cdot 4$ \\
\hline 3 & $1 \cdot 5515$ & - & 0.9749 & $1 \cdot 0921$ & $\begin{array}{l}1 \cdot 4641 \\
1 \cdot 448^{\mathrm{d}}\end{array}$ & $1 \cdot 0162$ & $111 \cdot 6$ & 108 & 118 & $-176 \cdot 8$ & $-67 \cdot 2$ \\
\hline $3 \mathbf{a}$ & $1 \cdot 5566$ & - & 0.9643 & $1 \cdot 1376$ & $\begin{array}{l}1 \cdot 4543 \\
1.4471^{\mathrm{d}}\end{array}$ & 1.003 & $111 \cdot 3$ & 104 & $115 \cdot 3$ & $-72 \cdot 8$ & $130 \cdot 7$ \\
\hline 4 & $1 \cdot 5106$ & - & - & $1 \cdot 0962$ & $\begin{array}{l}1 \cdot 269^{\mathrm{c}} \\
1.4495^{\mathrm{d}}\end{array}$ & - & $109 \cdot 6$ & - & $119 \cdot 2$ & $177 \cdot 7$ & $178 \cdot 8$ \\
\hline $4 a$ & 1.5147 & - & - & $1 \cdot 0918$ & $\begin{array}{l}1 \cdot 2727^{\mathrm{c}}, \\
1 \cdot 4447^{\mathrm{d}}\end{array}$ & - & $112 \cdot 2$ & - & $118 \cdot 2$ & $86 \cdot 7$ & 172.9 \\
\hline 5 & $1 \cdot 5155$ & - & - & $1 \cdot 1209$ & $\begin{array}{l}1 \cdot 3032^{\mathrm{c}} \\
1.455^{\mathrm{d}}\end{array}$ & $1 \cdot 0173$ & $109 \cdot 2$ & - & $124 \cdot 2$ & $-165 \cdot 1$ & -178 \\
\hline 6 & $1 \cdot 3461^{\mathrm{a}}$ & $1 \cdot 3785$ & 0.9764 & 1.0849 & $\begin{array}{l}1.4167 \\
1.4503^{\mathrm{d}}\end{array}$ & $1 \cdot 0154$ & 124 & - & $117 \cdot 3$ & $-102 \cdot 7$ & $113 \cdot 4$ \\
\hline $6 a$ & $1 \cdot 3565^{\mathrm{a}}$ & $1 \cdot 3497$ & 1.05 & $1 \cdot 0876$ & $\begin{array}{l}1.4226 \\
1.4755^{\mathrm{d}}\end{array}$ & 1.0203 & $122 \cdot 7$ & - & $115 \cdot 6$ & $-96 \cdot 1$ & 93.9 \\
\hline 7 & $1 \cdot 5125$ & $1 \cdot 2245^{\mathrm{b}}$ & - & $\begin{array}{l}1 \cdot 0989 \\
1 \cdot 1113\end{array}$ & $\begin{array}{l}1 \cdot 4461 \\
1.4456\end{array}$ & 1.0166 & $116 \cdot 6$ & - & $115 \cdot 5$ & $158 \cdot 7$ & $164 \cdot 2$ \\
\hline $7 \mathbf{a}$ & $1 \cdot 4765$ & $1 \cdot 2389^{\mathrm{b}}$ & - & $\begin{array}{l}1 \cdot 101 \\
1 \cdot 1287\end{array}$ & $\begin{array}{l}1.4528 \\
1.4762^{\mathrm{d}}\end{array}$ & $1 \cdot 02$ & $117 \cdot 1$ & - & $111 \cdot 4$ & $140 \cdot 7$ & $162 \cdot 8$ \\
\hline
\end{tabular}

${ }^{\mathrm{a}} \mathrm{C}=\mathrm{C} ;{ }^{\mathrm{b}} \mathrm{C}=\mathrm{O} ;{ }^{\mathrm{c}} \mathrm{N}=\mathrm{C} ;{ }^{\mathrm{d}} \mathrm{N}-\mathrm{C}\left(\mathbf{Y} / \mathbf{Y}^{-}\right) ;{ }^{\mathrm{e}} \mathrm{C}-\mathrm{N}-\mathrm{C}\left(\mathbf{Y} / \mathbf{Y}^{-}\right) ;{ }^{\mathrm{f}} \mathrm{C}-\mathrm{C}-\mathrm{C}-\mathrm{C}(\mathbf{X}) ;{ }^{\mathrm{g}} \mathrm{C}-\mathrm{C}-\mathrm{N}-\mathrm{C}\left(\mathbf{Y} / \mathbf{Y}^{-}\right)$

under basic conditions in the gaseous phase interstellar medium is higher than that of neutral conditions. In c-Rib/Rib + UGly reaction, 7 contains the lowest $\mu_{\text {Gas }}$, whereas $7 \mathbf{a}$ and $\mathbf{6 a}$ contain the highest and second highest $\mu_{\text {Gas }}$ in c-Rib/Rib + DGly reaction (table 1). $\Delta H_{\mathrm{f}}^{\circ}$ for 7 is found as the second highest in c-Rib/Rib + UGly reaction, and the second and third lowest for $\mathbf{6 a}$ and $7 \mathbf{a}$, respectively, in c$\mathbf{R i b} / \mathbf{R i b}+$ DGly reaction (table 1 ).

\subsection{Kinetics}

While the main purpose of this work is to propose thermochemical mechanisms for the formation of
Maillard products in the interstellar medium (ISM) we can also analyse the kinetics of certain paths. Several hundred transition states were attempted to be localized however, we were only able to identify five mechanisms presented here. In reality, many of the ISM reactions occur via barrier-less reactions ${ }^{7}$ and can proceed via three body interactions. The transition states that are most probable to be located are for c-Rib to Rib, 3 to 4, 6 to 7, 2a to 3a, and 4a to 6a transformations (figures 1 and 2). To perform these computations, we have verified the B3LYP/ 6-31G* frequencies to make sure that the imaginary frequency correspond to the correct reaction route. 

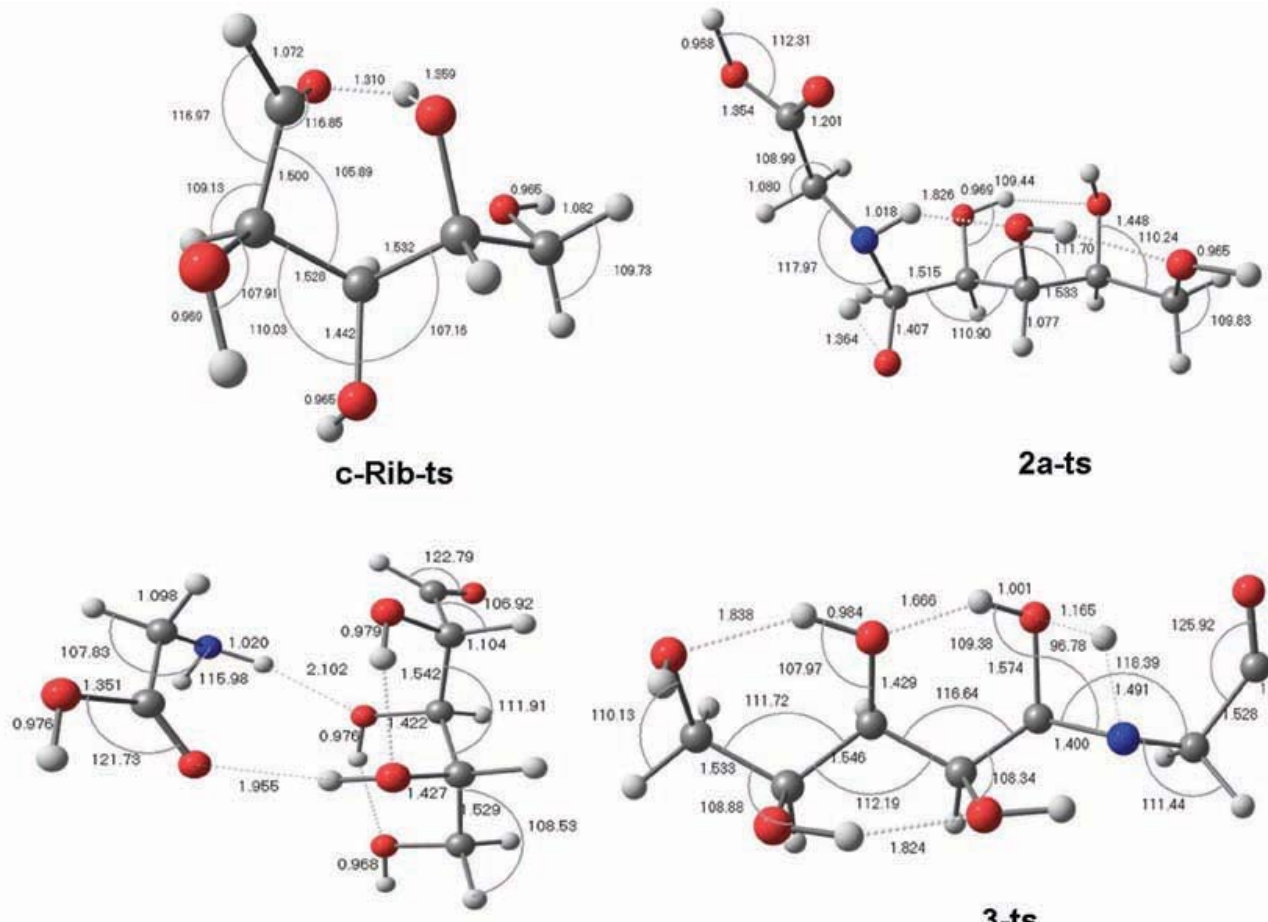

2-ts'

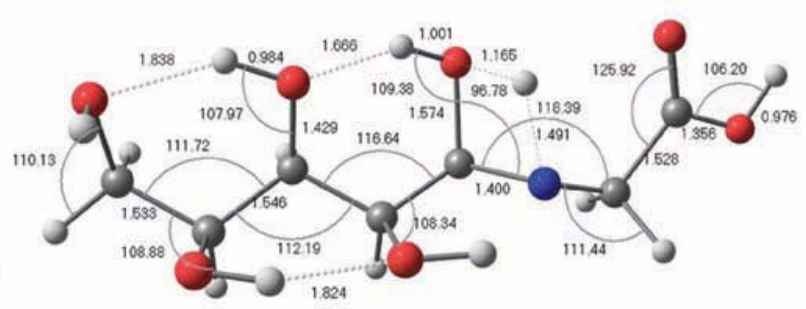

3-ts
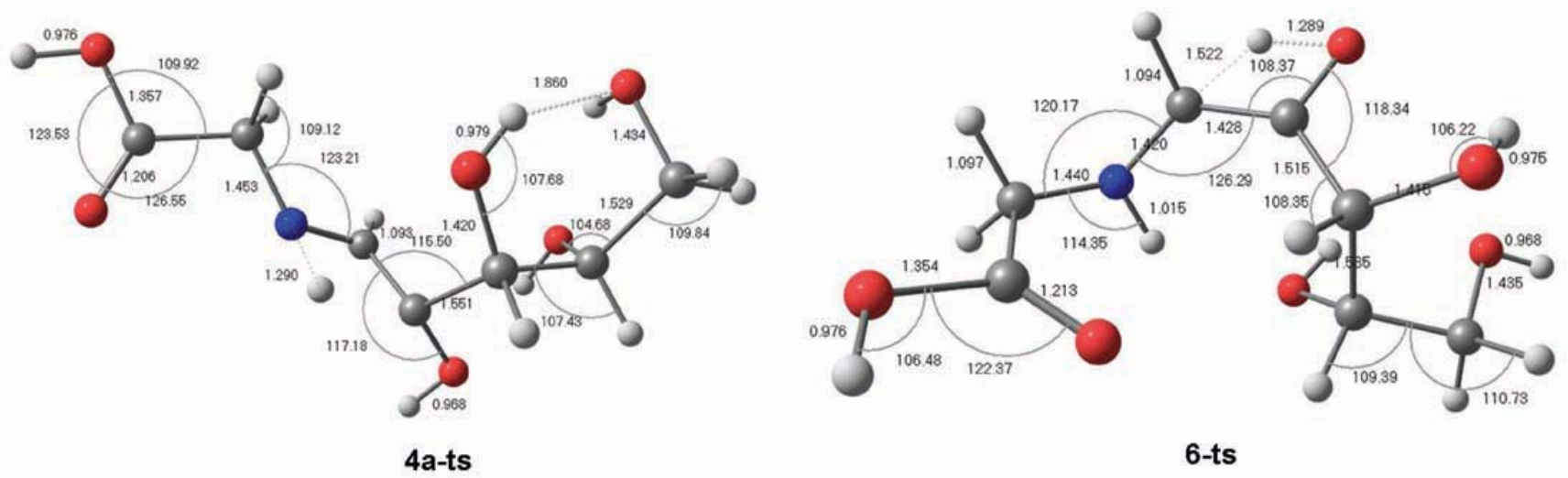

6-ts

Figure 3. Transition state structures for the species depicted for species labelled in table 3 where selected geometrical parameters are shown. Note that bond angles are in degrees $(\cdot)$ and bond lengths in angstroms $(\AA)$.

We have calculated the reaction rates using conventional transition state theory $(\mathrm{CTST})^{23}$ where the rate has the following form:

$$
k(T)=\left(k_{B} T / h c^{0}\right) e[-\Delta G / R T] .
$$

In this equation $k_{B}$ is the Boltmanz constant, $T$ is temperature, $c^{0}$ is the concentration (set to 1 ), $R$ is the ideal gas constant, and $\Delta G$ is the free energy of activation. Figure 3 shows transition state structures for the species depicted for species labelled in table 3 where selected geometrical parameters are shown.
Additionally, to evaluate the free energy of activation $\left(E_{a}\right)$ we have used the following equation:

$$
\ln k=\ln A-E_{a} / R T,
$$

whereby the slope of the equation corresponds to $-E_{a} / R$ from which the activation energy can be extracted. This equation was used to define an accurate activation energy based on kinetic factors which is important when analysing ISM behaviour.

Table 3 shows The $\Delta E_{0}$ values which are the forward batter heights (with zero-point effects inclu- 
Table 3. Various thermodynamic and kinetic parameters for the transition state mechanisms shown in figure 3 . The $E_{a}$ is the kinetically derived energies of activation used to describe the processes shown. The $\Delta E_{0}$ values are the forward barrier heights (with zeropoint effects included), $\Delta H$ is the enthalpy of activation, $\Delta G$ is the free energy of activation in $\mathrm{kcal} / \mathrm{mol}$ and $k\left(\mathrm{~s}^{-1}\right)$ is the reaction rate constant. Also shown are the imaginary frequencies that correspond to the mode of the reaction pathway following for the mechanism under consideration.

\begin{tabular}{lcccccc}
\hline Transition state & $v\left(\mathrm{~cm}^{-1}\right)$ & $E_{\mathrm{a}}(\mathrm{kJ} / \mathrm{mol})$ & $\Delta E_{0}(\mathrm{~kJ} / \mathrm{mol})$ & $\Delta H_{298}(\mathrm{~kJ} / \mathrm{mol})$ & $\Delta G_{298}(\mathrm{~kJ} / \mathrm{mol})$ & $k\left(\mathrm{~s}^{-1}\right) \times 10^{-3}$ \\
\hline c-Rib-ts & $1762 \mathrm{i}$ & $57 \cdot 40$ & $159 \cdot 26$ & $159 \cdot 55$ & $158 \cdot 67$ & $0 \cdot 2125$ \\
2a-ts & $1592 \mathrm{i}$ & $50 \cdot 55$ & 136.88 & $131 \cdot 44$ & $147 \cdot 14$ & $3 \cdot 315$ \\
2'-ts & $1605 \mathrm{i}$ & $82 \cdot 13$ & $192 \cdot 00$ & $194 \cdot 43$ & $200 \cdot 11$ & $1 \cdot 052 \times 10^{-5}$ \\
3-ts & $1576 \mathrm{i}$ & $58 \cdot 94$ & $153 \cdot 23$ & $148 \cdot 33$ & $161 \cdot 21$ & $0 \cdot 1147$ \\
4a-ts & $1929 \mathrm{i}$ & $119 \cdot 87$ & $266 \cdot 81$ & $266 \cdot 35$ & $263 \cdot 43$ & $2 \cdot 815 \times 10^{-12}$ \\
6-ts & $2227 \mathrm{i}$ & $115 \cdot 46$ & 243.03 & $249 \cdot 15$ & $256 \cdot 02$ & $1 \cdot 654 \times 10^{-11}$ \\
\hline
\end{tabular}

ded), $\Delta H$ is the enthalpy of activation, $\Delta G$ is the free energy of activation in $\mathrm{kcal} / \mathrm{mol}$ and $\mathrm{k}\left(\mathrm{s}^{-1}\right)$ is the reaction rate constant. Additionally, the imaginary frequency corresponding to the reaction pathway being described has also been displayed. These were used to confirm the assignment that a particular transition state corresponds to a reaction pathway being analysed.

For the first pathway we can see that the ribose isomerization (c-Rib $\rightarrow$ Rib) has a forward barrier height of around $159 \mathrm{~kJ} / \mathrm{mol}$, a free energy of activation $\left(E_{\mathrm{a}}\right)$ of $57.4 \mathrm{~kJ} / \mathrm{mol}$ and a rate of $0.2125 \times$ $10^{-3} \mathrm{~s}^{-1}$. While the rate of the reaction is a bit slow, under the conditions of the ISM this should occur with at least some frequency. The next step (2a to 3a) which has been described in figures $1-2$ has a free energy of activation of $147.14 \mathrm{~kJ} / \mathrm{mol}$ with a much smaller reaction rate constant. This can be attributed to the high polarity of the amino group which prefers the $\mathbf{3 a}$ configuration. This should be definitely possible in the ISM under various adverse conditions and when collisions are taken into consideration. Therefore, if this is the case, possibly this mechanism can proceed via a barrier-less process. Additionally, the energy of reaction suggests that this step is indeed favourable. It is our belief that this pathway can be of direct relevance to chemistry in the ISM. We do believe that this process can occur in the ISM.

A competing mechanism is the formation of a hydrogen bonded complex which is slightly higher in energy and has a free energy of activation of $200 \cdot 11 \mathrm{~kJ} / \mathrm{mol}$ (via $2^{\prime}$-ts shown in figure 3) with a small reaction rate. The activation energy for this process is $50.55 \mathrm{~kJ} / \mathrm{mol}$ which is still within the margin of error for these calculations to be considered viable in the ISM. While this is a potential mechanism the concentration of this product should be minimized in an experimental setting. We have presented this result but it is likely that it will not form under typical conditions.

Once $\mathbf{2}$ is formed from the basic reactions, $\mathbf{3}$ can form from a change in configuration via the transition state shown in figure 3 . As we can see the free energy of activation is $161.2 \mathrm{~kJ} / \mathrm{mol}$ with a higher reaction rate and therefore it should form experimentally. The first product 2a has a reaction rate which is about twice as low and should be preferred over structure 4 (which occurs through the transition state labelled as 3-ts is shown in figure 3). The $E_{a}$ value of this reaction is $58.94 \mathrm{~kJ} / \mathrm{mol}$ which is still feasible and can take place in the ISM. Test calculations with a larger B3LYP/6-311++G** basis set yield a value of $43.52 \mathrm{~kJ} / \mathrm{mol}$ that suggests that this reaction is highly favourable.

The final two isomerization steps (4a-ts $\rightarrow \mathbf{6 a} ; \mathbf{6}-$ ts $\rightarrow 7$ ) are high energy processes with slow reaction rates. The forward barrier heights are elevated, but the activation energies $\left(E_{a}\right)$ suggest that these reactions may not be feasible in the ISM but are a possibility. However, as we will show later the latter step to these products may indeed be barrier-less and not involve a transition state. It is another potential possibility that an external molecule can participate to protonate a structure leading to different chemical structures by external catalysts. Alternatively, the protonation steps can lead to the barrier-height through 4a-ts and 6-ts to be reduced since this process involves a high energy hydrogen migration. These steps may be feasible however, from an analysis of the activation energies even within the margin of error they may not occur.

These protonation and deprotonation processes were not completely modelled since we believe these steps may proceeed intermolecularly. The preliminary calculations suggest that the energies shown 
are not favourable and other mechanisms should be preferred. The calculation on the KET step may also be problematic due to uncertainty in the mechanism. The intramolecular 1,3-H shift transition state was located however, the intermolecular KET process will proceed more easily with the help of another reactant, solvent, or other possible proton donor and acceptor, as reported by previous studies. This will be the focus of a future study with respect to proton catalysed mechanisms of the reactions studied here.

\section{Conclusions}

It is clear from the present study that the Maillard reaction between c-Rib/Rib and Gly species may take place in the gaseous phase in the interstellar medium. c-Rib is more efficient than Rib in the reaction. The basic and neutral conditions are the first and second most favourable for the formation of ARPs. Formation of the enol form of ARP is not feasible under neutral condition, whereas the basic condition facilitates the formation of both of the enol and keto forms of ARP.

Therefore, the rate of the reaction is assumed higher under basic conditions than that of the neutral conditions. Based on our kinetics calculations we have shown that the conversion of $\mathbf{c}-\mathbf{R i b}$ to $\mathbf{R i b}$ is feasible along with $\mathbf{2 a} \rightarrow \mathbf{3 a}$ and $\mathbf{3} \rightarrow \mathbf{4}$. Other transition state mechanisms were not able to be located easily and the pathways discussed are the most probable routes for ISM molecule formation. Experimentally, these short lived products can be located by the imaginary frequencies presented and should be able to act as a guide for ISM molecule scans. Other competing reaction paths are probably feasible but this report serves to shed light on potential Maillard reactions in the ISM. These questions are of definite interest to a vast majority of chemists and physicists trying to understand the mistry of origin of life. The primary purpose of this work was to provide thermochemical data supporting this mechanism as a plausible factor for biochemistry in the ISM. However, our brief kinetics study can be of some use. It serves to show that the primary compounds exhibited should form at least in some concentrations under diverse conditions.

From the proposed calculations we have shown that in all cases the energies of reaction are indeed exothermic revealing that the processes that are undergone should be favourable from an energetic standpoint. Single point CCSD(T)/aug-cc-pVQZ calculations (with zero-point effects included) reveal that the barrier-heights through all pathways except for 4a-ts and 6-ts are feasible. Within the margin of error for theoretical calculations of transition states and activation barriers (which are in the order of 45$55 \mathrm{~kJ} / \mathrm{mol}$ ) that suggests that potentially these are barrier-less processes.

Our future focus will be on how we can apply these experimental and theoretical methods to investigate the possible formation of ribose and higher sugars. Additionally, the presence of free molecular oxygen in the formose reaction must be examined. Many researchers ${ }^{24-25}$ have shown that oxygen can have a definite influence on the reactivity of larger molecular systems. Particularly in steps involving $\mathrm{CO}$ groups its seems to favour the formation of oxygen based compounds. However, this is still tentative and needs to be further proved by experimental and theoretical evaluations. Structural determinations based on our experimental set-up will be the area of further investigations from our groups. Few studies that exist investigate the potential formation of ribose and related biomolecules in the ISM. Nevertheless, it is important to be aware that, while these products have not been observed, scientists must not be reluctant to pursue this area of research.

What must be noted is that the energies of reaction are highly favourable for the mechanisms proposed, therefore potentially speaking there are two options to explain this effect. The first is that another lower energy transition state may exist experimentally or the reaction is indeed barrier-less that will occur due to three-body collisions with external molecules. Also, the calculated imaginary frequencies for the transition states can be used in the spectroscopic determination of the relative strengths of a particular reaction pathway. If the frequencies are known that researchers can gear studies aimed at investigating regions of the vibrational spectra in the efforts to locate interesting ISM molecules. The transition states that were not able to locate by our methods probably correspond to processes or reactions that are barrier-less. These barrier-less mechanisms are common in the ISM and can be used to explain the presence of rare molecular species. It is important to note that future studies by our group include the theoretical analysis of external catalyst species that can help for certain chemical mechanisms to occur. However, this work should serve as an important first step in the proposal for the origin of interstellar life. 
Under acidic conditions and at the isoelectric point of glycine, the reaction is supposed to be the least and second least favourable. Therefore, the rate of the reaction under these conditions is assumed to be hindered. The theoretical results, presented in this paper, would be helpful for further theoretical and experimental investigations, leading to find out the real possibility of the Maillard reaction to take place in the interstellar medium and the relation of the reaction to the creation of first living organisms.

\section{Acknowledgements}

The Universidad Nacional Autonoma de Mexico (UNAM) is thanked for valuable financial and computational support. The authors thank anonymous reviewer for useful comments in the revision of this manuscript.

\section{References}

1. Maillard L C 1912 C.R. Acad. Sci. Ser. 14566

2. Davídek J, Velíšek J and Pokorný J 1990 Development in food science: Chemical changes during food processing (Amsterdam: Elsevier Science Publishers) Vol. 21

3. Eskin N A E 2000 Biochemistry of foods (San Diego: Academic Press, Inc.) 2nd edn

4. Macrane R, Robinson R K and Saadler M J 1993 Encyclopedia of food science, food technology and nutrition (London: Academic Press Limited) Vol. 1

5. Ledl F and Schleicher E 1990 Angew. Chem. Int. Ed. Engl. 29565

6. Zubay G 1999 Chemtracts 12432
7. Jalbout A F, Abrell L M, Adamowicz L, Polt R and Ziurys L M 2007 Astrobiol. J. 7 433; Jalbout A F and Shipar M D 2008 Origin Life Evol. Biosph. (in press)

8. Ricardo A, Carrigan M A, Olcott A N and Benner S A 2004 Science 303196

9. Larralde R, Robertson M P and Miller S L 1995 Proc. Natl. Acad. Sci. USA 928158

10. Breslow R 1959 Tet. Lett. 2122

11. Halfen D T, Apponi A, Woolf N, Polt R and Ziurys L M 2006 Astrophys. J. 639237

12. Chyba C F, Thomas P J, Brookshaw L and Sagan C 1990 Science 249366

13. Kuan, Y-J, Charnley S B, Huang H-C, Tseng W-L and Kisiel Z 2003 Astrophys. J. 593848

14. Snyder L E, Lovas F J, Hollis J M, Friedel D N, Jewell P R, Remijan A, Ilyushin V V, Alekseev E A and Dyubko S F 2004 Astrophys. J. 619914

15. Blagojevic V, Petrie S and Bohme D K 2003 Mon. Not. R. Astr. Soc. 339 L7

16. Jackson D M, Stibrich N J, McLain, J L, Fondren L D, Adams N G and Babcock L M 2005 Int. J. Mass Spectrom. 24755

17. Hodge J E 1953 J. Agric. Food Chem. 1928

18. Holum J R 1996 Introduction to organic and biological chemistry (New York: John Wiley \& Sons Inc)

19. Harrold H 1991 Organic chemistry: A short course (Boston: Houghton Mifelin Company) 8th edn

20. Frisch M J et al 2001 Gaussian 98, Revision A.11.2, Gaussian, Inc., Pittsburgh PA

21. Foresman J B and Frisch A E 1996 Exploring chemistry with electronic structure methods (Gaussian, Inc., Pittsburgh PA) 2nd edn

22. Hansel A, Singary W, Wisthaler A, Schwarzmann M and Lindinger W 1997 Intl. J. Mass Spectrom. Ion Proc. 167/168 1955

23. Jalbout A F, Chang C M, Galano A and Solimannejad M 2004 J. Mol. Struct. (THEOCHEM) 676127

24. Jalbout A F Origin Life Evol. Biosph. (in press)

25. Osamura Y, Fukuzawa K, Terzieva R and Herbst E 1999 Astrophys. J. 519697 